\title{
Association of genetic polymorphisms in genes involved in Ara-C and dNTP metabolism pathway with chemosensitivity and prognosis of adult acute myeloid leukemia (AML)
}

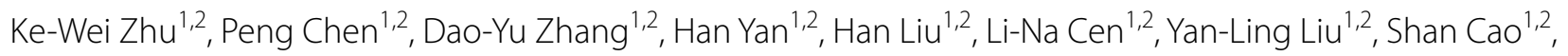
Gan Zhou ${ }^{1,2}$, Hui Zeng ${ }^{4}$, Shu-Ping Chen ${ }^{4}$, Xie-Lan Zhao ${ }^{4}$ and Xiao-Ping Chen ${ }^{1,2,3,5^{*}}$

\begin{abstract}
Background: Cytarabine arabinoside (Ara-C) has been the core of chemotherapy for adult acute myeloid leukemia (AML). Ara-C undergoes a three-step phosphorylation into the active metabolite Ara-C triphosphosphate (ara-CTP). Several enzymes are involved directly or indirectly in either the formation or detoxification of ara-CTP.

Methods: A total of 12 eQTL (expression Quantitative Trait Loci) single nucleotide polymorphisms (SNPs) or tag SNPs in 7 genes including CMPK1, NME1, NME2, RRM1, RRM2, SAMHD1 and E2F1 were genotyped in 361 Chinese non-M3 AML patients by using the Sequenom Massarray system. Association of the SNPs with complete remission (CR) rate after Ara-C based induction therapy, relapse-free survival (RFS) and overall survival (OS) were analyzed.

Results: Three SNPs were observed to be associated increased risk of chemoresistance indicated by CR rate (NME2 rs3744660, E2F1 rs3213150, and RRM2 rs1130609), among which two (rs3744660 and rs1130609) were eQTL. Combined genotypes based on E2F1 rs3213150 and RRM2 rs 130609 polymorphisms further increased the risk of non-CR. The SAMHD1 eQTL polymorphism rs6102991 showed decreased risk of non-CR marginally $(P=0.055)$. Three SNPS (NME1 rs3760468 and rs2302254, and NME2 rs3744660) were associated with worse RFS, and the RRM2 rs 1130609 polymorphism was marginally associated with worse RFS $(P=0.085)$ and OS $(P=0.080)$. Three SNPS (NME1 rs3760468, NME2 rs3744660, and RRM1 rs183484) were associated with worse OS in AML patients.
\end{abstract}

Conclusion: Data from our study demonstrated that SNPs in Ara-C and dNTP metabolic pathway predict chemosensitivity and prognosis of AML patients in China.

Keywords: Adult acute myeloid leukaemia (AML), Cytarabine arabinoside (Ara-C), Single nucleotide polymorphisms (SNPs)

\footnotetext{
*Correspondence: chenxp74@hotmail.com

${ }^{5}$ Department of Clinical Pharmacology, Xiangya Hospital, Central South

University, Changsha 410008, Hunan, China

Full list of author information is available at the end of the article
} 


\section{Background}

Acute myeloid leukemia (AML) is a hematological malignancy characterized by malignant proliferation of the hemopoietic system. AML is a heterogeneous collection of diseases characterized by distinct morphological, chromosomal and cytogenetic abnormalities. Subtype specific therapy for AML is not available despite the FAB M3 subtype. Cytarabine arabinoside (Ara-C) remains the first-line chemotherapeutic agent for the treatment of AML for decades [1-3]. Clinical studies have demonstrated that the complete remission (CR) rate ranges in $50-70 \%$ and 5 year survival rate ranges in $27-40 \%$ in AML patients receiving Ara-C based chemotherapy [4-6].

Ara-C is a nucleotide analog that is transmembrane transported into leukemia cells by the nucleoside transporters include the solute carrier family 29 member 1 (SLC29A1) [7]. Intracellular Ara-C undergoes a threestep phosphorylation into the activate metabolite Ara-C triphosphate (ara-CTP) by deoxycytidine kinase (DCK) [8], cytidine monophosphate kinase 1 (CMPK1) [9], and nucleoside diphosphate kinases (NDPKs) in turn [10]. Ara-CTP competes with deoxycytidine triphosphate (dCTP) for incorporation into DNA, and thus results in blockade of DNA synthesis and cell death [11]. More recently, Schneider $C$ and colleagues found that araCTP is hydrolyzed by the deoxynucleoside triphosphate
(dNTP) triphosphohydrolase SAM domain and HD domain 1 (SAMHD1), which promotes the detoxification of intracellular ara-CTP pools [12]. Disruption of $S A M H D 1$ increases Ara-C sensitivity in both cultured leukemia cells and mouse model of AML [13].

Intracellular level of dNTPs, which are consisted of dCTP, dATP, dTTP and dGTP, is another potential mechanism that affect Ara-C sensitivity. High intracellular dNTPs can inhibit Ara-C phosphorylation and decrease the accumulation of ara-CTP through inhibition of $D C K$ activity by a feedback mechanism. Exhaustion of the intracellular CTP/dCTP pools facilitates Ara-C phosphorylation and increases the incorporation of ara-CTP into DNA by reducing the feedback inhibition on $D C K$ [14]. Ribonucleotide reductase (RR) is a key enzyme responsible for the reduction of ribonucleotides to deoxyribonucleotides and plays important roles in the regulation of intracellular CTP/dCTP pools [15, 16] (Fig. 1). RR is mainly composed of RRM1 and RRM2 subunits, and the expression of $R R M 2$ is regulated by the transcription factor E2F1 [17]. An inverse correlation between $R R M 1 / R R M 2$ mRNA expression and intracellular araCTP in leukemia blasts after Ara-C treatment is observed in clinic. Ex vivo ara-C sensitivity study with primary AML samples has also shown correlationship between RRM1 mRNA expression and Ara-C sensitivity [18]. In addition, higher $R R M 1$ expression in leukemia blast

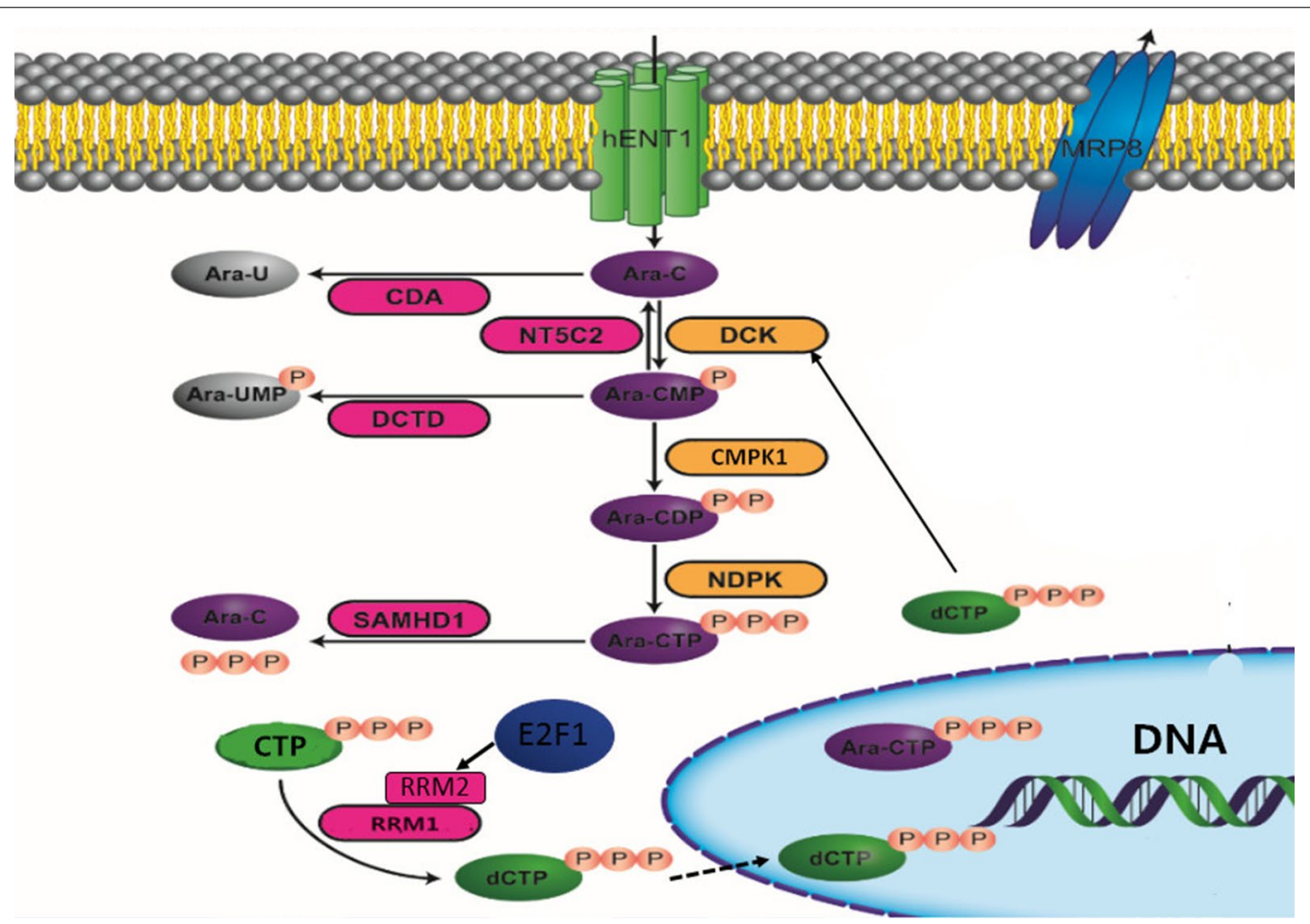

Fig. 1 Schematic diagram of the Ara-C metabolizing pathway 
cells predicts better relapse-free survival (RFS) in AML patients [19].

Influence of genetic factors on either Ara-C chemosensitivity and/or AML prognosis has raised much interest in recent years $[20,21]$. Our previous studies have shown associations of single nucleotide polymorphisms (SNPs) in DCK, Wilms tumor 1 (WT1) and DNA methyltransferase 3 alpha $(D N M T 3 A)$ with drug response to Ara-C based induction therapy in AML [22-24]. In addition, somatic mutations in genes such as DNMT3A, FMS-like Tyrosine Kinase3 (FLT3) and nucleophosmin (NPM1) can also affect AML prognosis [24-26]. For the Ara-C metabolic pathway genes, a promoter polymorphism rs2302254 in NME1 (encoding NDPK-A) is associated increased risk of Ara-C associated neurotoxicity [27]. The RRM1 3'-UTR SNP rs1042919 and promoter SNP rs1561876 are reported to be associated with decreased intracellular ara-CTP levels during Ara-C therapy, and increased risk of relapse and/or worse event free survival (EFS) in Caucasian AML patients treated with cytarabine and cladribine [18]. More recently, association of RRM1 rs9937 variant with induction therapy related death and survival rates in AML patients is also reported [20]. In the study by $\mathrm{Cao}$ et al. the $R R M 2$ nonsynonymous SNP rs1130609 (S59A) is associated with decreased Ara-C cytotoxicity in HapMap lymphoblast cell lines from the CEU population and poorer EFS for the St Jude AML97 cohort, but the clinical association was not replicated in St Jude AML02 cohort [18]. There is no studies focused on the association of CMPK1, NME2, SAMHD1 and E2F1 polymorphisms with Ara-C response in AML patients presently.

In this study, we sought to identify genetics polymorphisms in Ara-C and dNTP metabolic pathway including CMPK1, NME1/NME2 (encoding NDPK), SAMHD1, $R R M 1, R R M 2$ and $E 2 F 1$ with drug response to Ara-C based chemotherapy and AML prognosis in Chinese AML patients.

\section{Methods \\ Patients}

Three hundred and sixty-one newly diagnosed AML patients were recruited from Xiangya hospital from May 2009 to Nov 2017. Peripheral venous blood or bone marrow samples were obtained before chemotherapy. Patients with AML FAB M3 subtype, or combined critical illness and cancer, less than 16 years old, and secondary leukemia were excluded. M3 subtype was excluded because this subtype of patients had unique chemotherapy regimens.

All patients received an " $7+3$ " induction regimen consisting of Ara-C (100-200 mg/m $\mathrm{m}^{2}$ for day 1-7) and anthracyclines (mitoxantrone $8-16 \mathrm{mg} / \mathrm{m}^{2}$, or daunorubicin $45-60 \mathrm{mg} / \mathrm{m}^{2}$, or aclarubicin $20 \mathrm{mg} / \mathrm{m}^{2}$, or pirarubicin $30 \mathrm{mg} / \mathrm{m}^{2}$, or idarubicin $10-20 \mathrm{mg} / \mathrm{m}^{2}$, for day 1-3). One or two courses of induction chemotherapy were given to obtain complete remission (CR). Achievement of CR was defined according to the international recommendations as we described previously [23], which include: $<5 \%$ blasts in the bone marrow; absence of extramedullary disease; absolute neutrophil count $>1.0 \times 10^{9} / \mathrm{L}$; platelet count $>100 \times 10^{9} / \mathrm{L}$. Once CR was achieved, the patients received sequential consolidation therapy consisting of Ara-C and anthracyclines or haematopoietic stem cell transplantation (HSCT). Regular follow-up was carried out by outpatient review or telephone.

The clinical and pathological information of the AML patients were obtained by chart review of electronic medical record (EMR) from the hospital. Overall survival (OS) and relapse free survival (RFS) were used to indicate disease outcomes. Relapse was defined as the presence of $>5 \%$ of blast cells in the bone marrow or reappearance of blast cells in the peripheral blood or development of extramedullary disease. RFS was calculated from the date of achievement of CR until the date of relapse or death from any cause. OS was calculated from the date of AML diagnosis to the date of death from any cause. For those patients without relapse or death event by the end of the study follow-up, survival end-points were censored at the date of last follow-up. The risk stratification criteria based on cytogenetics and molecular characteristics was described elsewhere [23].

\section{SNPs selection and genotyping}

Based on literature searching on Ara-C metabolism related genes in the PubMed database, 7 genes involved in Ara-C metabolic pathway (CMPK1, NEM1, NEM2, $S A M H D 1, R R M 1, R R M 2, E 2 F 1)$ were selected. Candidate tag SNPs in these genes were initially selected based on the database from NCBI (https://www.ncbi.nlm.nih.gov/ snp/) and the 1000 Genomes Project (https://www.ncbi. nlm.nih.gov/variation/tools/1000 genomes/) using the Haploview 4.2 (Cambridge, MA, USA). Then, potential influence of the SNPs on expression the corresponding genes was analyzed by the expression quantitative trait locus (eQTL) database (https://gtexportal.org/home/). A total of 12 SNPs (CMPK1 rs7543016, NME1 rs3760468 and rs2302254, NME2 rs3744660, SMAD1 rs6102991, rs28372906 and rs6029941, E2F1 rs3213150 and rs3213180, RRM1 rs2412344 and rs183484, and RRM2 rs1130609) were finally selected (Table 1, Fig. 2).

Genomic DNA was extracted from blood samples using Blood DNA Kit II (Omega Bio-Tek, USA) according to the manufacturer's protocol. The 12 candidate SNPs were genotyped by ionization-time-of-flight mass spectrometry (Sequenom, SanDiego, CA). The genotyping success 
Table 1 Candidate SNPs selected in the study and influence on gene expression according to eQTL database

\begin{tabular}{|c|c|c|c|c|c|c|}
\hline SNP & Gene & Position & MAF & Alleles & Region & eQTL $P$ \\
\hline rs7543016 & CMPK1 & 1:47333967 & 0.467 & $C / G$ & Gly8Arg & 0.3 \\
\hline rs3760468 & NME1 & 17:51153130 & 0.376 & $\mathrm{~A} / \mathrm{T}$ & Promoter & $2.3 e^{-21}$ \\
\hline rs2302254 & NME1 & 17:51153539 & 0.087 & $C / T$ & Promoter & $2.7 e^{-13}$ \\
\hline rs3744660 & NME2 & 17:51168540 & 0.162 & $\mathrm{~A} / \mathrm{G}$ & Intron & $2.5 e^{-12}$ \\
\hline rs6102991 & SAMHD1 & 20:36956238 & 0.386 & $\mathrm{~A} / \mathrm{G}$ & Promoter & $2.0 e^{-6}$ \\
\hline rs28372906 & SAMHD1 & 20:36951753 & 0.063 & $C / T$ & 5'-UTR & NA \\
\hline rs6029941 & SAMHD1 & 20:36891027 & 0.447 & $A / G$ & $3^{\prime}-$ UTR & $2.1 e^{-8}$ \\
\hline rs3213150 & $E 2 F 1$ & $20: 33687284$ & 0.357 & $C / T$ & Intron & NA \\
\hline rs3213180 & E2F1 & 20:33675818 & 0.286 & $C / G$ & $3^{\prime}-$ UTR & NA \\
\hline rs2412344 & RRM1 & $11: 4086354$ & 0.430 & $\mathrm{C} / \mathrm{T}$ & Promoter & $1.1 e^{-6}$ \\
\hline rs183484 & RRM1 & 11:4119902 & 0.381 & $\mathrm{G} / \mathrm{T}$ & Arg284Arg & $9.4 \mathrm{e}^{-6}$ \\
\hline rs1130609 & RRM2 & 2:10122793 & 0.343 & $\mathrm{G} / \mathrm{T}$ & 5'-UTR/Ser59Ala & $1.2 e^{-7}$ \\
\hline
\end{tabular}

$N A$ expression data is not available in the eQTL database

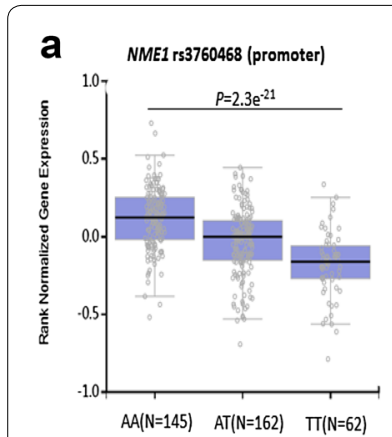

e SAMHD 1 rs6102991 (promoter)

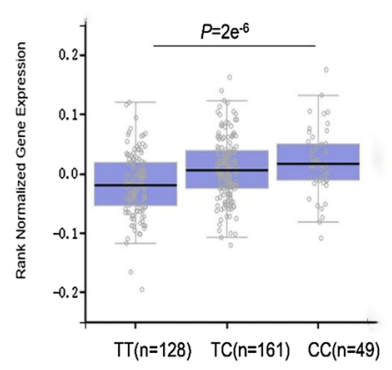

b NME1 rs2302254 (promoter)
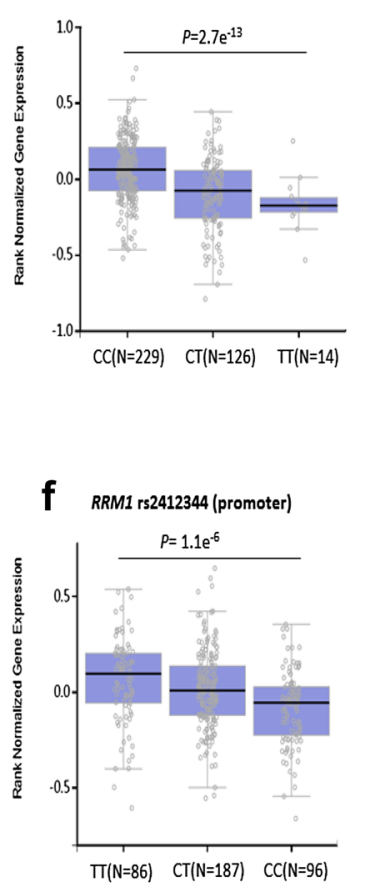

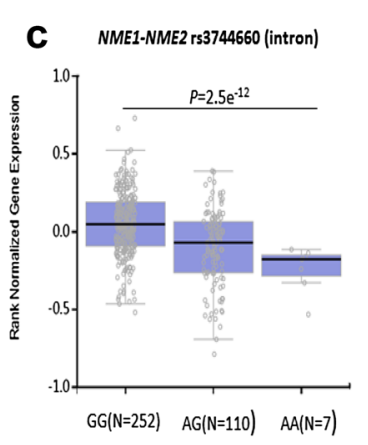

d
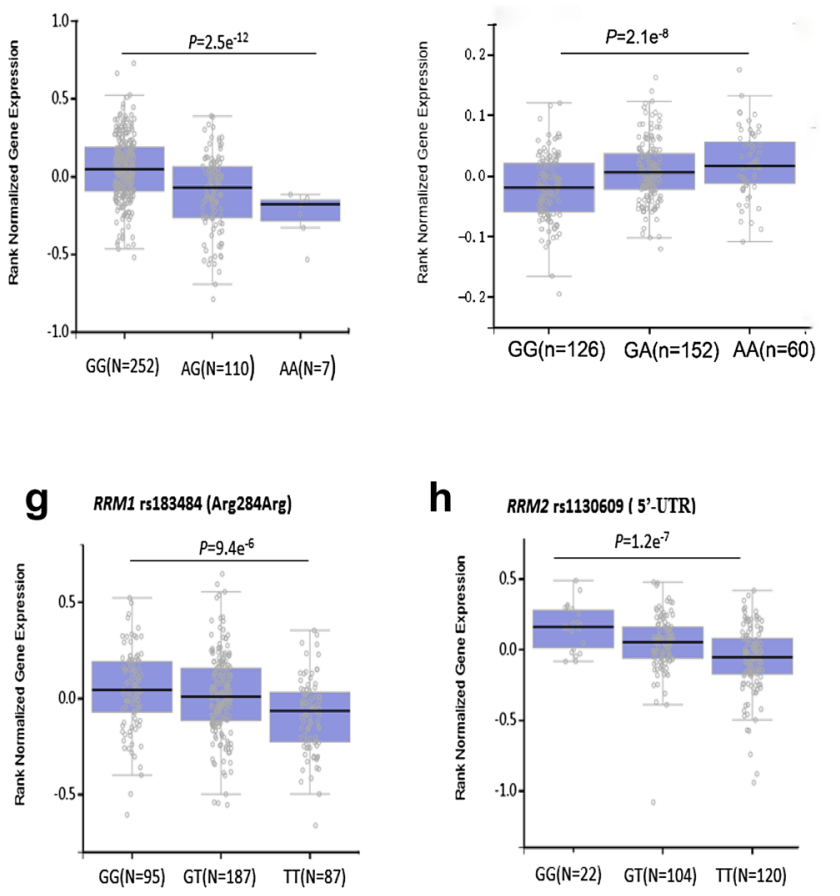

h

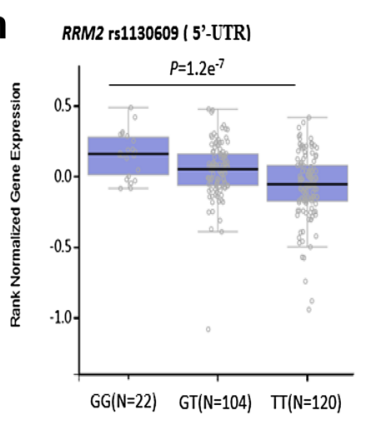

Fig. 2 eQTL analysis of 8 candidate SNPs selected in the study. a NME1 rs3760468; b NME1 rs2302254; c NME1-NME2 rs3744660; d SAMHD1 rs6029941; e SAMHD1 rs6102991; f RRM1 rs2412344; g RRM1 rs183484; h RRM2 rs1130609

rates were higher than $96.4 \%$ except for the rs7543016 polymorphism $(89.8 \%)$ by the Massarray system. Genotyping results for the SNPs were confirmed in 10\% randomly selected samples by PCR-based sequencing.

\section{Statistical analysis}

Statistical analyses were carried out with the IBM SPSS software (version 22.0; IBM SPSS Inc., Chicago, IL, USA).
Comparisons of continuous variables between genotype groups were performed by nonparametric test (MannWhitney $U$ test). Fitness of the genotype distribution to Hardy-Weinberg Equilibrium (HWE) was analyzed by using the $X^{2}$ test. Associations of genotypes with CR or non-CR was analyzed by unconditional logistic regression and indicated by odds ratio (OR) and the $95 \%$ confidence interval (CI). Survival probabilities were estimated 
by the Kaplan-Meier method, and differences between genotypes were evaluated using the log-rank test. Cox proportional hazard models were constructed for RFS and OS, adjusting for potential confounding covariates including gender, age at diagnosis, risk stratification, alloSCT, WBC count, serum lactate dehydrogenase (LDH) level and bone marrow blast counts at diagnosis as necessary. A stepwise selection method was used to determine the potential confounding covariates. Hazard ratio (HR) was used to estimate association of risk factors with RFS and OS. Three genetic models (dominant, recessive and additive model) were tested for the genotype association analysis. $P<0.05$ was considered to be significant.

\section{Results}

\section{Baseline characteristics and the overall CR status of the AML patients}

The baseline clinical characteristics of the 361 AML patients were summarized in Table 2. The median age of the patients was 43 [range 16-76] years old. Among the AML patients, 196 were male and 165 were female. According to FAB classification, the patients were classified into 7 subtypes. M2 was the most common subtype (53.5\%), followed by M5 (20.8\%), and no patient with the M7 or M0 subtype was recruited. The median number of WBC was $14.7 \times 10^{9} / \mathrm{L}$, the mean serum level of $\mathrm{LDH}$ was $363 \mathrm{U} / \mathrm{L}$. Other clinical information was also shown in Table 2. When stratified by cytogenetics and molecular abnormalities, 71,187 , and 78 patients were classified as low risk, intermediate risk, and high risk, respectively. Information of karyotype and somatic mutations was not available for 25 patients. For the 361 patients received Ara-C based induction therapy, 211 patients (58.4\%) received Ara-C+mitoxantrone (MA) or Ara-C+idarubicin (IA) regimens, 47 patients (13.0\%) received Ara-C + THP (TA), 52 patients (14.4\%) received Ara-C + aclarubicin + G-CSF (CAG) and 31 patients (8.6\%) received Ara-C+daunorubicin (DA). A total of 205 patients $(56.8 \%)$ achieved CR after one or two courses of chemotherapy, and CR could not be evaluated accurately for $36(10.0 \%)$ patients due to insufficiencies in clinical evidence. Sixty-four (17.7\%) patients received hematopoietic stem cell transplantation, 178 patients died during the follow-up time, and 7 (1.9\%) patients were out of touch after several follow-up. The median and mean follow-up period for OS analysis were 926 and 1235 days, respectively.

Comparison of CR rate among genotypes after Ara-C based induction therapy and results of unconditional logistic regression analysis

Significant differences in $C R$ rates among genotypes for the NME2 rs3744660 $(P=0.047), E 2 F 1$ rs3213150
Table 2 The baseline characteristics of 316 AML patients

\begin{tabular}{|c|c|c|}
\hline Characteristics & Totality, n (\%) & Median (range) \\
\hline Gender & 361 & \\
\hline Male & $196(54.3 \%)$ & \\
\hline Female & $165(45.7 \%)$ & \\
\hline Age & 361 & $43(16-76)$ years \\
\hline \multicolumn{3}{|l|}{ FAB classification } \\
\hline M1 & $19(5.3 \%)$ & \\
\hline M2 & $193(53.5 \%)$ & \\
\hline M4 & $63(17.5 \%)$ & \\
\hline M5 & 75 (20.8\%) & \\
\hline M6 & $6(1.7 \%)$ & \\
\hline Undefined & $5(1.4 \%)$ & \\
\hline WBC & $356(98.6 \%)$ & $14.7(0.4-426.0), \times 10^{9} / \mathrm{L}$ \\
\hline $\mathrm{LDH}$ & $341(94.5 \%)$ & $363(17-5853), \mathrm{U} / \mathrm{L}$ \\
\hline BM blast \% & $348(96.4 \%)$ & $71 \%(17-99 \%)$ \\
\hline $\mathrm{RBC}$ & $356(98.6 \%)$ & $2.19(0.67-4.98), \times 10^{12} / \mathrm{L}$ \\
\hline Hemoglobin & $356(98.6 \%)$ & $72(27-149), g / L$ \\
\hline Platelets & $356(98.6 \%)$ & $34(2-546), \times 10^{9} / \mathrm{L}$ \\
\hline Neutrophil & $356(98.6 \%)$ & $2.2(0.0-340.3), \times 10^{9} / \mathrm{L}$ \\
\hline \multicolumn{3}{|l|}{ Risk stratifications } \\
\hline Low risk & $71(19.7 \%)$ & \\
\hline Intermediate risk & $187(51.8 \%)$ & \\
\hline High risk & $78(21.6 \%)$ & \\
\hline Undefined & $25(6.9 \%)$ & \\
\hline \multicolumn{3}{|l|}{ FLT3-ITD mutation } \\
\hline Positive & $42(11.6 \%)$ & \\
\hline Negative & $294(81.4 \%)$ & \\
\hline Unknown & $25(6.9 \%)$ & \\
\hline \multicolumn{3}{|l|}{ NPM1 mutation } \\
\hline Positive & $67(18.6 \%)$ & \\
\hline Negative & $269(74.5 \%)$ & \\
\hline Unknown & $25(6.9 \%)$ & \\
\hline \multicolumn{3}{|l|}{ CEBPA mutation } \\
\hline Positive & $50(13.9 \%)$ & \\
\hline Negative & $286(79.2 \%)$ & \\
\hline Unknown & $25(6.9 \%)$ & \\
\hline \multicolumn{3}{|l|}{ Karyotype } \\
\hline Normal & $255(70.6 \%)$ & \\
\hline Abnormal & $84(23.3 \%)$ & \\
\hline Unknown & $22(6.1 \%)$ & \\
\hline \multicolumn{3}{|l|}{ Allo-HCT } \\
\hline Yes & $64(17.7 \%)$ & \\
\hline No & $297(82.3 \%)$ & \\
\hline \multicolumn{3}{|c|}{ CR after two courses of induction therapy } \\
\hline Yes & $205(56.8 \%)$ & \\
\hline No & $120(33.2 \%)$ & \\
\hline Not evaluated & $36(10.0 \%)$ & \\
\hline \multicolumn{3}{|c|}{ Chemotherapy regimens } \\
\hline MA & $125(34.6 \%)$ & \\
\hline IA & $86(23.8 \%)$ & \\
\hline TA & $47(13.0 \%)$ & \\
\hline
\end{tabular}


Table 2 (continued)

\begin{tabular}{lll}
\hline Characteristics & Totality, $\mathbf{n}(\%)$ & Median (range) \\
\hline DA & $31(8.6 \%)$ \\
CAG & $52(14.4 \%)$ \\
Other regimens & $20(5.5 \%)$ \\
\hline
\end{tabular}

$F A B$ French-Britain-American, $W B C$ white blood cell, $L D H$ lactate dehydrogenase, $B M$ bone marrow, $R B C$ red blood cell, Allo-SCT allogeneic hematopoietic stem cell transplantation, $C R$ complete remission, THP pirarubicin, MA Ara-C + mitoxantrone, IA Ara-C+idarubicin, TA Ara-C+THP, DA Ara-C + daunorubicin, CAG Ara-C + aclarubicin + G-CSF

$(P=0.043)$, and $R R M 2$ rs1130609 $(P=0.042)$ polymorphisms were observed ( $p$ values from Chi square test by three genotype groups). For the NME2 rs3744660 polymorphism, the CR rate was $68.5,53.9$, and $57.1 \%$, respectively, for the GG, GA and AA genotypes, and carriers of the rs3744660 A allele (dominant model) showed significantly increased non-CR (indicative of chemoresistance) rate (GA+AA vs GG, $45.6 \%$ vs $31.5 \%$, crude $\mathrm{OR}=1.829, \quad 95 \%$ CI $1.127-2.967, \quad P=0.014$ ) (Table 3). For the E2F1 rs3213150 polymorphism, the CR rate was $60.4,69.2$, and $50.0 \%$, respectively, for the CC, CT, and TT genotypes, and rs3213150 TT homozygous showed significantly increased non-CR rate as compared with carriers of the rs3213150 $\mathrm{C}$ allele (recessive model) $(50.0 \%$ vs $34.4 \%$, crude OR $=1.903$, $95 \%$ CI $1.014-3.574, P=0.043)$. For the $R R M 2$ rs1130609 polymorphism, the CR rate was 64.1, 67.1, and $44.1 \%$, respectively, for the TT, TG and GG genotypes, and patients with the rare GG genotype showed significantly increased non-CR rate as compared with carriers of the $\mathrm{T}$ allele (GG vs TT+TG, $55.9 \%$ vs $34.3 \%$, crude OR $=2.428,95 \%$ CI 1.181-4.990, $P=0.014)$. Carriers of the $S A M H D 1$ rs6102991 G allele (GA+GG) also showed marginally decreased non-CR rate as compared with the wild-type AA homozygotes (crude OR $=0.611$, 95\% CI 0.369-1.013, $P=0.055)$. No differences in CR rates among genotypes for other SNPs were observed (Table 3).

Logistic regression analysis showed significant associations of risk stratification, age, pretreatment WBC counts and LDH levels with non-CR risk (Additional file 1: Table S1). When adjusted by these risk factors, NME2 rs3744660, E2F1 rs3213150 and RRM2 rs1130609 were still significantly associated with risk of non-CR (Table 3). NME2 rs3744660 AG genotype or carriers of the rs3744660 A allele showed increased risk for non-CR (AG vs GG: adjusted OR $=1.953,95 \%$ CI 1.092-3.484, $P=0.024 ; A G+A A$ vs $G G$ : adjusted $\mathrm{OR}=1.852$, 95\% CI $1.062-3.236, P=0.030)$. For the E2F1 rs3213150 polymorphism, as compared with patients carrying the E2F1 rs3213150 C allele, those with the TT genotype showed increased risk of non-CR (adjusted OR $=2.208,95 \%$ CI $1.065-4.567, P=0.033$ ). While for the RRM2 rs1130609 polymorphism, the rare GG homozygotes showed significantly increased risk of non-CR in comparison with carriers of the $\mathrm{T}$ allele (adjusted OR $=2.398,95 \%$ CI 1.025-5.618, $P=0.044$ ). Marginally decreased risk of non-CR was also observed for the $S A M H D 1$ rs6102991 polymorphism in a dominant model (GA+GG vs AA, adjusted OR $=0.611,95 \%$ CI 0.349-1.071, $P=0.086)$.

\section{Combined influence of E2F1 and RRM2 polymorphisms on chemosensitivity to Ara-C based induction therapy in AML patients}

As E2F1 is a transcription factor involved in the regulation of RRM2 expression, a combined genotype analysis of the E2F1 and RRM2 polymorphisms on CR rate was carried out. As both E2F1 rs3213150 and RRM2 rs1130609 polymorphisms were associated with increased risk for non-CR in negative models, patients were grouped based on genotypes of the two SNPs (Table 5): rs1130609 TT+GT/rs3213150 CC+CT (both favorable for $\mathrm{CR}, \mathrm{n}=237)$, rs1130609 TT+GT/rs3213150 TT, n=37), rs1130609 GG/rs3213150 CC+CT (n=27), and rs1130609 GG/rs3213150 TT (both unfavorable for $\mathrm{CR}, \mathrm{n}=6)$. Significant difference in CR rate was observed among the four groups $\left(\chi^{2}=10.637, P=0.014\right)$, which was 66.7, 54.1, 48.1, and 16.7\%, respectively (Table 5). As compared with the favorable genotypes combination group (rs1130609 TT+GT/rs3213150 CC+CT), carriers of two unfavorable genotypes (rs1130609 GG/rs3213150 TT) and those carrying at least one unfavorable genotype showed significantly increased risk of non-CR $(\mathrm{OR}=10.00,95 \%$ CI 1.149-87.06, $P=0.004 ; \mathrm{OR}=2.118$, 95 CI 1.233-3.637, $P=0.006$, respectively, Table 4). After adjustment for age, risk stratification, and WBC counts, results of logistic regression analysis indicated that patients carrying both or at least one unfavorable genotype showed significantly increased risk of non-CR $(\mathrm{OR}=9.780,95 \%$ CI 1.099-87.069, $P=0.041$; OR $=2.257$, 95 CI 1.273-4.002, $P=0.005$, respectively, Table 4).

\section{Influence of candidate SNPs on RFS and OS for AML patients}

Univariate analysis showed significant differences in RFS among genotypes of NME1 rs3760468, NME1 rs2302254, and NME2 rs3744660 (Table 5, Fig. 3). All the three SNPs were associated with worse RFS in a recessive model (rs3760468 TT vs AA+AT: $\mathrm{HR}=1.752$, 95\% CI $1.142-$ 2.686, $P=0.009$; rs2302254 TT vs $\mathrm{CC}+\mathrm{CT}: \mathrm{HR}=1.912$, 95\% CI $1.117-3.272, \quad P=0.016 ; \quad$ rs3744660 AA vs GG+AG: $H R=2.087,95 \%$ CI $1.051-4.145, P=0.036)$. 
Table 3 Comparison of CR rate among genotypes after 2 courses of Ara-C based chemotherapy

\begin{tabular}{|c|c|c|c|c|c|c|c|}
\hline Genotype & Total (n) & CR, n (\%) & Non-CR, n (\%) & $P$ & OR $(95 \% \mathrm{Cl})$ & $P^{a}$ & OR $(95 \% \mathrm{Cl})^{\mathrm{a}}$ \\
\hline \multicolumn{8}{|c|}{ CMPK1 rs7543016 } \\
\hline $\mathrm{CC}$ & 93 & $57(61.3 \%)$ & $36(38.7 \%)$ & & 1.00 (reference) & & 1.00 (reference) \\
\hline GC & 167 & $106(63.5 \%)$ & $61(36.5 \%)$ & 0.727 & $0.911(0.540-1.537)$ & 0.921 & $0.970(0.531-1.772)$ \\
\hline GG & 30 & $23(76.7 \%)$ & 7 (23.3\%) & 0.125 & $0.482(0.188-1.238)$ & 0.076 & $0.365(0.120-1.111)$ \\
\hline \multicolumn{8}{|c|}{ NME1 rs3760468 } \\
\hline $\mathrm{AA}$ & 115 & $77(67.0 \%)$ & 38 (33.0\%) & & 1.00 (reference) & & 1.00 (reference) \\
\hline AT & 154 & $96(62.3 \%)$ & $58(37.7 \%)$ & 0.434 & $1.224(0.737-2.033)$ & 0.256 & $1.420(0.775-2.597)$ \\
\hline $\mathrm{TT}$ & 44 & $26(59.1 \%)$ & $18(40.9 \%)$ & 0.353 & $1.403(0.686-2.869)$ & 0.465 & $1.359(0.597-3.096)$ \\
\hline \multicolumn{8}{|c|}{ NME1 rs 2302254} \\
\hline CC & 186 & $123(66.1 \%)$ & $63(33.9 \%)$ & & 1.00 (reference) & & 1.00 (reference) \\
\hline CT & 114 & $65(57.0 \%)$ & $49(43.0 \%)$ & 0.114 & $1.473(0.912-2.375)$ & 0.288 & $1.355(0.775-2.370)$ \\
\hline $\mathrm{TT}$ & 19 & $13(68.4 \%)$ & $6(31.6 \%)$ & 0.840 & $0.901(0.327-2.481)$ & 0.534 & $0.688(0.212-2.232)$ \\
\hline \multicolumn{8}{|c|}{ NME2 rs3744660 } \\
\hline GG & 213 & $146(68.5 \%)$ & $67(31.5 \%)$ & & 1.00 (reference) & & 1.00 (reference) \\
\hline$A G$ & 89 & $48(53.9 \%)$ & $41(46.1 \%)$ & 0.016 & $1.861(1.121-3.091)$ & 0.024 & $1.953(1.092-3.484)$ \\
\hline AA & 14 & $8(57.1 \%)$ & $6(42.9 \%)$ & 0.376 & $1.634(0.545-4.897)$ & 0.770 & $1.299(0.361-4.673)$ \\
\hline $\mathrm{AA}+\mathrm{AG}$ & 103 & $56(54.4 \%)$ & $47(45.6 \%)$ & 0.014 & $1.829(1.127-2.967)$ & 0.030 & $1.852(1.062-3.236)$ \\
\hline \multicolumn{8}{|c|}{ SAMHD1 rs6102991 } \\
\hline AA & 88 & $49(55.7 \%)$ & $39(44.3 \%)$ & & 1.00 (reference) & & 1.00 (reference) \\
\hline GA & 161 & $107(66.5 \%)$ & $54(33.5 \%)$ & 0.093 & $0.634(0.372-1.080)$ & 0.183 & $0.669(0.370-1.209)$ \\
\hline GG & 62 & $43(69.4 \%)$ & $19(30.6 \%)$ & 0.090 & $0.555(0.280-1.101)$ & 0.063 & $0.483(0.224-1.038)$ \\
\hline $\mathrm{AG}+\mathrm{GG}$ & 223 & $150(67.3 \%)$ & $73(32.7 \%)$ & 0.055 & $0.611(0.369-1.013)$ & 0.086 & $0.611(0.349-1.071)$ \\
\hline \multicolumn{8}{|c|}{ SAMHD1 rs28372906 } \\
\hline $\mathrm{TT}$ & 284 & $180(63.4 \%)$ & $104(36.6 \%)$ & & 1.00 (reference) & & 1.00 (reference) \\
\hline CT & 32 & $21(65.6 \%)$ & $11(34.4 \%)$ & 0.802 & 0.907 (0.420-1.955) & 0.878 & $0.935(0.394-2.217)$ \\
\hline $\mathrm{CC}$ & 4 & $3(75.0 \%)$ & $1(25.0 \%)$ & 0.632 & $0.577(0.059-5.618)$ & 0.953 & $0.933(0.091-9.524)$ \\
\hline \multicolumn{8}{|c|}{ SAMHD1 rs6029941 } \\
\hline GG & 88 & $50(56.8 \%)$ & $38(43.2 \%)$ & & 1.00 (reference) & & 1.00 (reference) \\
\hline GA & 161 & $107(66.5 \%)$ & $54(33.5 \%)$ & 0.132 & 0.664 (0.389-1.132) & 0.339 & $0.739(0.398-1.374)$ \\
\hline $\mathrm{AA}$ & 65 & $42(64.6 \%)$ & $23(35.4 \%)$ & 0.330 & $0.721(0.372-1.395)$ & 0.370 & $0.706(0.330-1.512)$ \\
\hline \multicolumn{8}{|c|}{ E2F1 rs3213180 } \\
\hline GG & 149 & $95(63.8 \%)$ & $54(36.2 \%)$ & & 1.00 (reference) & & 1.00 (reference) \\
\hline GC & 141 & $90(63.8 \%)$ & $51(36.2 \%)$ & 0.990 & $0.997(0.617-1.610)$ & 0.915 & $0.970(0.552-1.704)$ \\
\hline CC & 29 & $16(55.2 \%)$ & $13(44.8 \%)$ & 0.383 & 1.429 (0.639-3.195) & 0.429 & $1.449(0.578-3.636)$ \\
\hline \multicolumn{8}{|c|}{ E2F1 rs3213150 } \\
\hline CC & 111 & $67(60.4 \%)$ & $44(39.6 \%)$ & & 1.00 (reference) & & 1.00 (reference) \\
\hline CT & 159 & $110(69.2 \%)$ & $49(30.8 \%)$ & 0.133 & $0.678(0.408-1.127)$ & 0.105 & $0.616(0.343-1.106)$ \\
\hline$\pi$ & 46 & $23(50.0 \%)$ & $23(50.0 \%)$ & 0.232 & $1.523(0.762-3.042)$ & 0.128 & $1.835(0.839-4.016)$ \\
\hline $\mathrm{CC}+\mathrm{CT}$ & 270 & $177(65.6 \%)$ & $93(34.4 \%)$ & & 1.00 (reference) & & 1.00 (reference) \\
\hline$T T$ & 46 & $23(50.0 \%)$ & $23(50.0 \%)$ & 0.043 & $1.903(1.014-3.574)$ & 0.033 & $2.208(1.065-4.567)$ \\
\hline \multicolumn{8}{|c|}{ RRM1 rs183484 } \\
\hline GG & 100 & $62(62.0 \%)$ & 38 (38.0\%) & & 1.00 (reference) & & 1.00 (reference) \\
\hline GT & 177 & $118(66.7 \%)$ & $59(33.3 \%)$ & 0.434 & $0.816(0.490-1.359)$ & 0.112 & $0.617(0.340-1.119)$ \\
\hline$T T$ & 42 & $22(52.4 \%)$ & $20(47.6 \%)$ & 0.287 & $1.483(0.716-3.071)$ & 0.263 & $1.616(0.697-3.731)$ \\
\hline \multicolumn{8}{|c|}{ RRM1 rs2412344 } \\
\hline$\pi$ & 89 & $56(62.9 \%)$ & $33(37.1 \%)$ & & 1.00 (reference) & & 1.00 (reference) \\
\hline CT & 183 & $119(65.0 \%)$ & $64(35.0 \%)$ & 0.734 & $0.913(0.539-1.545)$ & 0.510 & $0.817(0.449-1.488)$ \\
\hline CC & 48 & $28(58.3 \%)$ & $20(41.7 \%)$ & 0.599 & $1.212(0.592-2.483)$ & 0.967 & $1.018(0.429-2.415)$ \\
\hline \multicolumn{8}{|c|}{ RRM2 rs1130609 } \\
\hline $\mathrm{TT}$ & 131 & $84(64.1 \%)$ & 47 (35.9\%) & & 1.00 (reference) & & 1.00 (reference) \\
\hline GT & 149 & $100(67.1 \%)$ & 49 (32.9\%) & 0.599 & $0.876(0.534-1.436)$ & 0.920 & $0.972(0.557-1.697)$ \\
\hline
\end{tabular}


Table 3 (continued)

\begin{tabular}{|c|c|c|c|c|c|c|c|}
\hline Genotype & Total (n) & CR, n (\%) & Non-CR, n (\%) & $P$ & OR $(95 \% \mathrm{Cl})$ & $P^{a}$ & OR $(95 \% \mathrm{CI})^{a}$ \\
\hline GG & 34 & $15(44.1 \%)$ & $19(55.9 \%)$ & 0.034 & $2.264(1.053-4.867)$ & 0.069 & $2.347(0.935-5.882)$ \\
\hline $\mathrm{TT}+\mathrm{GT}$ & 280 & $184(65.7 \%)$ & $96(34.3 \%)$ & & 1.00 (reference) & & 1.00 (reference) \\
\hline GG & 34 & $15(44.1 \%)$ & $19(55.9 \%)$ & 0.014 & $2.428(1.181-4.990)$ & 0.044 & $2.398(1.025-5.618)$ \\
\hline
\end{tabular}

a Adjusted by age, risk stratification, WBC count, and serum LDH level

Table 4 Combined genotypes of RRM2 and E2F1 polymorphisms and chemotherapy sensitivity in AML patients

\begin{tabular}{|c|c|c|c|c|c|c|c|}
\hline rs1130609 & rs3213150 & CR, n (\%) & Non-CR, n (\%) & $P$ & OR $(95 \% \mathrm{Cl})$ & $P^{\mathrm{b}}$ & OR $(95 \% \mathrm{Cl})^{\mathrm{b}}$ \\
\hline $\mathrm{TT}+\mathrm{GT}$ & $\mathrm{CC}+\mathrm{CT}$ & $158(66.7 \%)$ & $79(33.3 \%)$ & & 1.00 (reference) & & 1.00 (reference) \\
\hline $\mathrm{TT}+\mathrm{GT}$ & $\mathrm{TT}$ & $20(54.1 \%)$ & $17(45.9 \%)$ & 0.134 & $1.700(0.844-3.426)$ & 0.048 & $2.115(1.006-4.450)$ \\
\hline GG & $\mathrm{CC}+\mathrm{CT}$ & $13(48.1 \%)$ & $14(51.9 \%)$ & 0.056 & $2.154(0.966-4.802)$ & 0.159 & $1.840(0.787-4.301)$ \\
\hline GG & $\mathrm{TT}$ & $1(16.7 \%)$ & $5(83.3 \%)$ & 0.004 & $10.00(1.149-87.06)$ & 0.041 & $9.780(1.099-87.069)$ \\
\hline \multicolumn{2}{|c|}{ Unfavorable combinations $^{a}$} & $34(48.6 \%)$ & $36(51.4 \%)$ & 0.006 & $2.118(1.233-3.637)$ & 0.005 & $2.257(1.273-4.002)$ \\
\hline
\end{tabular}

a Unfavorable combination genotypes included rs1130609 GG genotype or rs3213150 TT genotype

b Adjusted by age, risk stratification, WBC count, and serum LDH level

Table 5 Univariate and multivariate Cox regression analysis of SNPs associated with AML relapse-free survival (RFS)

\begin{tabular}{|c|c|c|c|c|c|c|c|}
\hline Genotype & $\mathbf{n}$ & Mean \pm SE (day) & Median (range, day) & $\mathrm{HR}(95 \% \mathrm{Cl})$ & $P$ & $\operatorname{HR}(95 \% \mathrm{Cl})^{\mathrm{a}}$ & $P^{a}$ \\
\hline \multicolumn{8}{|c|}{ NME1 rs3760468 } \\
\hline $\mathrm{AA}$ & 113 & $1058 \pm 202$ & 751 (294-1208) & 1.00 (reference) & & 1.00 (reference) & \\
\hline AT & 121 & $1163 \pm 212$ & $894(378-1410)$ & $0.946(0.657-1.362)$ & 0.764 & 1.027 (0.709-1.488) & 0.887 \\
\hline $\mathrm{TT}$ & 39 & $655 \pm 207$ & $357(285-429)$ & $1.703(1.070-2.709)$ & 0.025 & $1.744(1.085-2.801)$ & 0.022 \\
\hline$A A+A T$ & 234 & $1110 \pm 149$ & 761 (379-1143) & 1.00 (reference) & & 1.00 (reference) & \\
\hline $\mathrm{TT}$ & 39 & $655 \pm 207$ & $357(285-429)$ & $1.752(1.142-2.686)$ & 0.009 & $1.854(1.197-2.871)$ & 0.006 \\
\hline \multicolumn{8}{|c|}{ NME1 rs2302254 } \\
\hline CC & 169 & $941 \pm 129$ & 724 (4484-964) & 1.00 (reference) & & 1.00 (reference) & \\
\hline $\mathrm{CT}$ & 87 & $1324 \pm 266$ & $1282(516-2048)$ & $0.770(0.526-1.126)$ & 0.177 & $0.728(0.494-1.071)$ & 0.107 \\
\hline $\mathrm{TT}$ & 22 & $581 \pm 253$ & $373(286-460)$ & $1.756(1.014-3.042)$ & 0.044 & $1.688(0.973-2.928)$ & 0.062 \\
\hline $\mathrm{CC}+\mathrm{CT}$ & 256 & $1111 \pm 156$ & $760(398-1122)$ & 1.00 (reference) & & 1.00 (reference) & \\
\hline $\mathrm{TT}$ & 22 & $581 \pm 253$ & $373(286-460)$ & $1.912(1.117-3.272)$ & 0.016 & $1.878(1.096-3.217)$ & 0.022 \\
\hline \multicolumn{8}{|c|}{ NME2 rs3744660 } \\
\hline GG & 191 & $987 \pm 124$ & 751 (397-1105) & 1.00 (reference) & & 1.00 (reference) & \\
\hline$A G$ & 71 & $1239 \pm 283$ & $976(186-1766)$ & $0.929(0.627-1.377)$ & 0.714 & $0.958(0.644-1.425)$ & 0.832 \\
\hline AA & 12 & $382 \pm 116$ & $373(95-651)$ & $2.087(1.051-4.145)$ & 0.036 & $2.224(1.114-4.444)$ & 0.024 \\
\hline $\mathrm{GG}+\mathrm{AG}$ & 262 & $1136 \pm 157$ & 760 (399-1121) & 1.00 (reference) & & 1.00 (reference) & \\
\hline $\mathrm{AA}$ & 12 & $382 \pm 116$ & $373(95-651)$ & $2.127(1.078-4.194)$ & 0.026 & $2.250(1.135-4.460)$ & 0.020 \\
\hline \multicolumn{8}{|c|}{ RRM2 rs1130609 } \\
\hline $\mathrm{TT}$ & 123 & $1126 \pm 208$ & 751 (368-1134) & 1.00 (reference) & & 1.00 (reference) & \\
\hline GT & 125 & $1129 \pm 216$ & 724 (209-1239) & $1.561(0.692-1.386)$ & 0.907 & $0.926(0.651-1.317)$ & 0.668 \\
\hline GG & 29 & $679 \pm 272$ & $371(200-542)$ & $1.561(0.900-2.707)$ & 0.113 & $1.437(0.825-2.501)$ & 0.200 \\
\hline $\mathrm{TT}+\mathrm{GT}$ & 248 & $1127 \pm 159$ & $729(381-1077)$ & 1.00 (reference) & & 1.00 (reference) & \\
\hline GG & 29 & $679 \pm 272$ & $371(200-542)$ & $1.577(0.935-2.660)$ & 0.085 & $1.493(0.882-2.528)$ & 0.136 \\
\hline
\end{tabular}

a Adjusted for risk stratification, WBC count, and allo-SCT

The RRM2 rs1130609 GG genotype also showed marginally worse RFS (GG vs TT+GT: $\mathrm{HR}=1.577,95 \% \mathrm{CI}$ $0.935-2.660, P=0.085)$.
Multivariate analysis indicated that risk stratification, age, allo-SCT, WBC counts and serum LDH levels were associated with RFS of AML patients (Additional 
Table 6 Univariate and multivariate Cox regression analysis of SNPs associated with AML overall survival (OS)

\begin{tabular}{|c|c|c|c|c|c|c|c|}
\hline Genotype & $\mathbf{n}$ & Mean \pm SE (day) & Median (range) (day) & $\mathrm{HR}(95 \% \mathrm{Cl})$ & $P$ & $\operatorname{HR}(95 \% \mathrm{Cl})^{\mathrm{a}}$ & $P^{\mathrm{a}}$ \\
\hline \multicolumn{8}{|c|}{ NME1 rs3760468 } \\
\hline $\mathrm{AA}$ & 135 & $1326 \pm 209$ & 1534 (947-2121) & 1.00 (reference) & & 1.00 (reference) & \\
\hline AT & 160 & $1232 \pm 218$ & 768 (388-1148) & $1.238(0.890-1.724)$ & 0.205 & $1.455(1.015-2.087)$ & 0.041 \\
\hline $\mathrm{TT}$ & 47 & $801 \pm 231$ & $513(398-638)$ & $1.652(1.067-2.557)$ & 0.024 & $1.702(1.072-2.704)$ & 0.024 \\
\hline $\mathrm{AT}+\mathrm{TT}$ & 207 & $1159 \pm 185$ & $663(442-884)$ & $1.328(0.973-1.812)$ & 0.073 & $1.518(1.082-2.129)$ & 0.016 \\
\hline \multicolumn{8}{|c|}{ NME2 rs3744660 } \\
\hline GG & 230 & $1283 \pm 168$ & 1159 (615-1703) & 1.00 (reference) & & 1.00 (reference) & \\
\hline$A G$ & 98 & $1175 \pm 252$ & $817(410-1224)$ & $1.283(0.924-1.780)$ & 0.137 & $1.181(0.831-1.679)$ & 0.353 \\
\hline AA & 15 & $442 \pm 149$ & $359(167-551)$ & $2.214(1.154-4.247)$ & 0.017 & $2.284(1.145-4.559)$ & 0.019 \\
\hline $\mathrm{GG}+\mathrm{AG}$ & 328 & $1261 \pm 143$ & $988(682-1294)$ & 1.00 (reference) & & 1.00 (reference) & \\
\hline $\mathrm{AA}$ & 15 & $442 \pm 149$ & $359(167-511)$ & $2.054(1.080-3.905)$ & 0.025 & $2.172(1.098-4.294)$ & 0.026 \\
\hline \multicolumn{8}{|c|}{ RRM1 rs183484 } \\
\hline GG & 109 & $940 \pm 167$ & $672(454-890)$ & 1.00 (reference) & & 1.00 (reference) & \\
\hline GT & 194 & $1506 \pm 202$ & 1749 (799-2699) & $0.692(0.496-0.964)$ & 0.030 & $0.599(0.413-0.868)$ & 0.007 \\
\hline $\mathrm{TT}$ & 46 & $993 \pm 305$ & $414(162-666)$ & $1.112(0.717-1.726)$ & 0.635 & $1.063(0.665-1.698)$ & 0.799 \\
\hline \multicolumn{8}{|c|}{ RRM2 rs1130609 } \\
\hline$\pi$ & 148 & $1243 \backslash \pm 201$ & $1025(426-1624)$ & 1.00 (reference) & & 1.00 (reference) & \\
\hline GT & 161 & $1380 \pm 209$ & $843(520-1166)$ & $0.988(0.719-1.357)$ & 0.941 & $1.025(0.746-1.408)$ & 0.881 \\
\hline GG & 36 & $753 \pm 248$ & $473(137-809)$ & $1.506(0.917-2.475)$ & 0.106 & $1.489(0.906-2.448)$ & 0.116 \\
\hline $\mathrm{TT}+\mathrm{GT}$ & 309 & $1297 \pm 153$ & 935 (645-1225) & 1.00 (reference) & & 1.00 (reference) & \\
\hline GG & 36 & $753 \pm 248$ & $473(137-809)$ & $1.516(0.948-2.422)$ & 0.080 & $1.471(0.920-2.352)$ & 0.107 \\
\hline
\end{tabular}

${ }^{a}$ Adjusted for risk stratification, WBC count, allo-SCT, age, and serum LDH level

file 2: Table S2). When adjusted by these risk factors, NME1 rs3760468 TT, NME1 rs2302254 TT, and NME2 rs3744660 GG genotypes were still associated with worse RFS (rs3760468 TT vs AA+AT: adjusted $\mathrm{HR}=1.854,95 \%$ CI 1.197-2.871, $P=0.006$; rs2302254 TT vs CC+CT: adjusted $\mathrm{HR}=1.878,95 \%$ CI 1.096-3.217, $P=0.022$; rs3744660 AA vs GG+AG: adjusted $\mathrm{HR}=2.250,95 \% \mathrm{CI}$ 1.135-4.460, $P=0.020$ ).

Results of univariate analysis showed that the NME1 rs3760468, NME2 rs3744660, and RRM1 rs183484 polymorphisms were associated with OS of AML patients significantly (Table 6, Fig 4). As compared with rs3760468 AA homozygotes, rs3760468 TT homozygotes showed significantly worse OS (TT vs AA, HR $=1.652$, 95\% CI 1.067-2.557, $P=0.024)$ and carriers of the rs3760468 T allele showed a trend of worse OS (TT+AT vs $\mathrm{AA}: \mathrm{HR}=1.328,95 \% \mathrm{CI} 0.973-1.812, P=0.073)$. For the rs3744660 polymorphisms, the rare AA homozygotes showed significantly worse OS $(\mathrm{HR}=2.214,95 \%$ CI $1.154-4.247, P=0.017)$. For the RRM1 rs183484 polymorphism, the GT heterozygotes showed significantly better OS than the wild-type GG homozygotes $(\mathrm{HR}=0.692$, 95\% CI 0.496-0.964, $P=0.030)$. Marginally significant difference in OS between the RRM2 rs1130609 GG and GT+TT genotypes was also observed $(\mathrm{HR}=1.516,95 \%$ CI 0.948-2.422, $P=0.080$, Table 6).
Results from multivariate analysis indicated that risk stratification, age, allo-SCT, WBC counts and serum LDH levels were associated with OS of AML patients (Additional file 2: Table S2). When adjusted by these OS risk factors, rs3760468, rs3744660, and rs183484 polymorphisms were still associated with OS risk. As compared with rs3760468 AA homozygotes, rs3760468 TT homozygotes or carriers of the rs3760468 $\mathrm{T}$ allele showed significantly worse OS (TT+AT vs AA: adjusted $\mathrm{HR}=1.518,95 \%$ CI $1.082-2.129, P=0.016)$. For the rs3744660 polymorphisms, the rare AA homozygotes showed significantly worse OS (adjusted $\mathrm{HR}=2.284$, 95\% CI 1.145-4.559, $P=0.019)$. For the RRM1 rs183484 polymorphism, the GT heterozygotes showed significantly longer OS than the wild-type GG homozygotes $(\mathrm{HR}=0.599,95 \%$ CI $0.413-0.868, P=0.007)$.

\section{Discussion}

In this study, we observed the associations of 12 SNPs in 7 genes involved in Ara-C and dNTP metabolic pathway with chemosensitivity to Ara-C based therapy as well as disease prognosis in Chinese AML patients. We observed that 3 SNPs including NME2 rs3744660 (dominant model), E2F1 rs3213150 (recessive model) and RRM2 rs1130609 (recessive model) were associated with increased risk for non-CR after Ara-C based 

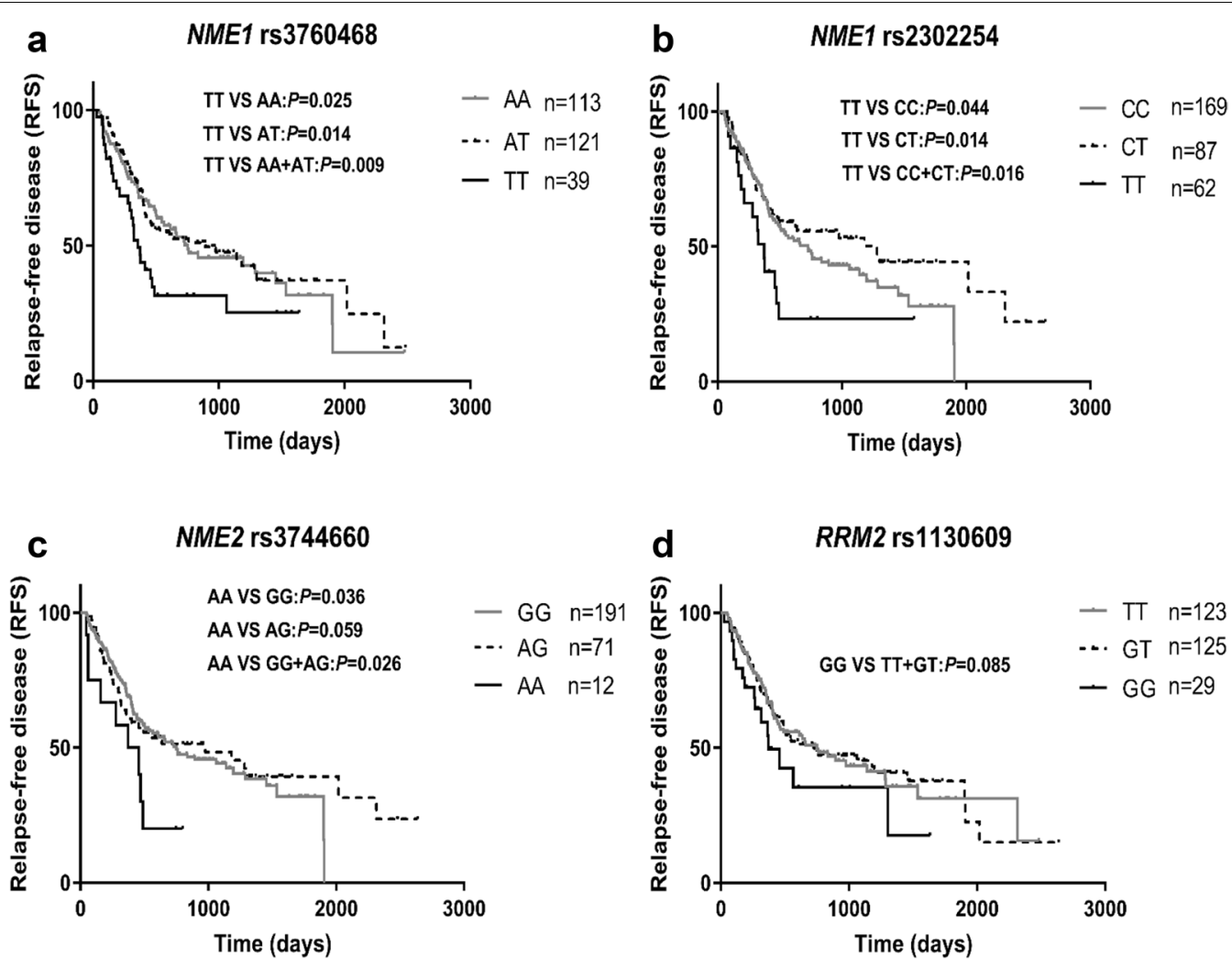

Fig. 3 Comparison of relapse-free survival (RFS) in AML patients among/between genotypes of NME1 rs3760468 (a); NME1 rs2302254 (b); NME2 rs3744660 (c); and RRM2 rs1130609 (d)

therapy, while the SAMHD1 rs6102991 polymorphism showed a tendency with decreased risk of non-CR. We also observed a combined effect of E2F1 and RRM2 polymorphisms on $C R$ rate. In addition, we observed that the NME1 rs3760468 and rs2302254, and NME2 rs3744660 polymorphisms predicted worse RFS, while NME1 rs3760468, NME2 rs3744660, and RRM1 rs183484 predicted worse OS in the AML patients.

For the 7 genes involved in our study, 3 genes (CMPK1, $N M E 1$ and $N M E 2$ ) were involved in the metabolic activation of Ara-C, while 4 genes (SAMHD1, E2F1, RRM1 and $R R M 2)$ were involved in the Ara-C inactivation or DCK feedback inhibition (dNTP/dCTP synthesis) pathways. Four genes (CMPK1, NME2, SAMHD1 and E2F1) were studied for the first time in our study. The majority of the SNPs included in our study were eQTL variants. For the 4 SNPs associated with CR rate observed in our study, difference in mRNA expression among genotypes of NME2 rs3744660 $\left(P=2.5 \mathrm{e}^{-12}\right), R R M 2 \operatorname{rs} 1130609\left(P=1.2 \mathrm{e}^{-7}\right)$, and $S A M H D 1 \mathrm{rs} 6102991\left(P=2.0 \mathrm{e}^{-6}\right)$ were indicated by eQTL analysis, and 2 of these SNPs (NME2 rs3744660, $S A M H D 1$ rs6102991) were reported for the first time in our study.
NME1 (alias DNPK-A) and NME2 (alias DNPK-B) are two most important members of the NDPK family of proteins and mediate phosphorylation of ara-CDP into ara-CTP. The coding genes NME1 and NME2 are closely neighbored on chromosome 17 . We observed that carriers of the NME2 intronic variant rs 3744660 was associated with decreased NME2 mRNA expression shown by eQTL analysis, which indicated decreased DNPK activity and ara-CTP formation, this is in agreement with the observation of decreased CR rate in patients carrying the rs3744660 A allele or worse prognosis in AA genotyped patients. Few studies have focused on NME1-NME2 locus presently. NME1 is initially identified for its metastatic suppressive potential for cancer cells [28]. Qu et al. reported that the NME1 promoter SNPs rs2302254 and rs3760468 can alter nuclear proteins binding capacity and reduce $N M E 1$ promoter activity by about $20 \%$, which may account for increased breast cancer mortality for these SNPs in Chinese [29]. In a study by Braunagel et al. the rs2302254 TT genotype (mutant homozygotes) was an independent risk factor for Ara-C associated neurotoxicity but not for OS or RFS in AML patients [26]. However, neither rs2302254 nor rs3760468 

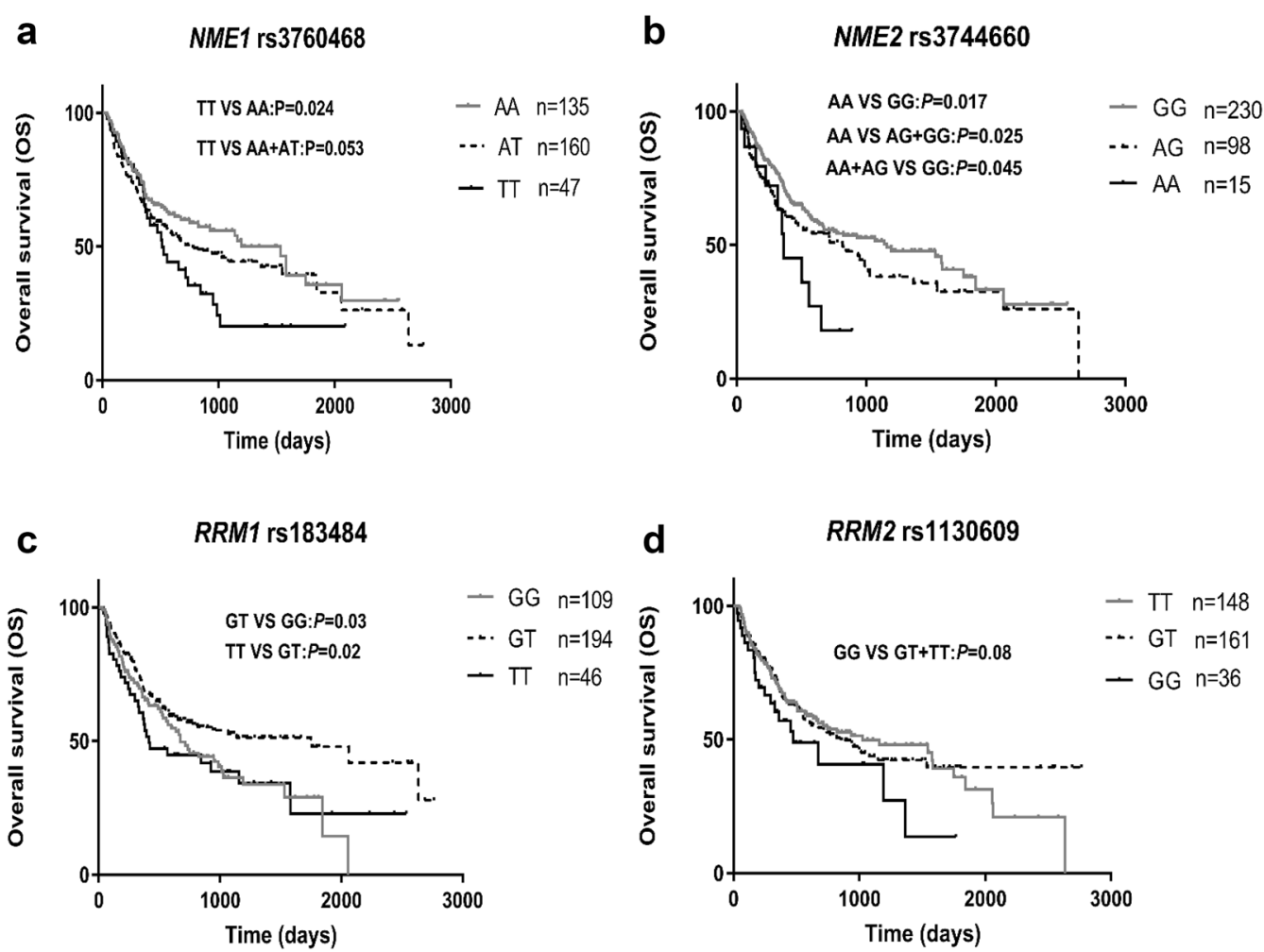

Fig. 4 Comparison of overall survival (OS) in AML patients among/between genotypes of NME1 rs3760468 (a); NME2 rs3744660 (b); RRM1 rs183484 (c); and RRM2 rs1130609 (d)

was associated with CR rate in our study, though both polymorphisms can results in decreased NME1 mRNA expression and predicted worse prognosis indicated by RFS and/or OS in our study. We assume that the positive findings in AML prognosis could also be explained, at least partially, by the direct functions of NME1/NME2, both can stimulate the growth and survival of AML cells through activation of the MAPK and transducers and activators of transcription signaling pathways [30, 31]. Of note, the rs3744660 is located in intron 4 of NME2 (NM_001018137.2) and intron 7 of the NME1-NME2 transcript (NM_001018136.2), the exact function of this SNP remains to be explored.

The RRM2 rs1130609 polymorphism is a non-synonymous SNP that leads to amino acid change from Ser59 to Ala59. We found that the rare GG genotype showed increased $R R M 2 \mathrm{mRNA}$ expression as indicated by eQTL analysis, which may suggest increased dNTP synthesis and increased feedback inhibition on $D C K$ activity in these patients. This is in accordance with the findings of increased non-CR risk and marginally worse prognosis in AML patients with the GG genotype in our study. In consistent with our study, higher RRM1/RRM2 mRNA expression was observed to be associated with decreased Ara-C sensitivity in the HapMap YRI samples (30 trios).
Our findings are also in agreement with a previous study by Cao et al. who reported that the rs1130609 polymorphism was associated with decreased Ara-C cytotoxicity in the HapMap CEU samples and worse EFS in Caucasian AML patient cohorts [18]. The exact function of the SNP remains unknown despite its influence on RRM2 mRNA expression. Search of the dbSNP database shows that the rs1130609 polymorphism is also a $5^{\prime}$-UTR SNP for the RRM2 transcript NM_001034.3. Further functional study of this SNP is required. Cao et al. reported significant associations of several RRM1 SNPs in the regulatory regions (e.g. rs2412344, rs11030907, rs7929397, rs2898950, rs1042919, and rs2412344) with ex vivo Ara-C cytotoxicity and/or in vivo Ara-C response [18]. A recent study in Chinese AML patients also observed association of the $3^{\prime}$-UTR potential miRNA binding site SNP rs1042919 with decreased CR rate but not disease prognosis in AML patients [32]. However, we failed to observed associations between the two RRM1 SNPs rs2412344 (in promoter) and rs183484 (Arg284Arg) and risk of non-CR, though significant differences in mRNA expression among the genotypes of both SNPs were indicated by eQTL analysis. We observed that the rs183484 GT genotype showed better OS $(\mathrm{HR}=0.599,95 \% \mathrm{CI}$ 
0.413-0.868, $P=0.007)$. The discrepancies among the studies should be further validated in future studies.

$E 2 F 1$ is a newly identified risk gene for Ara-C response in our study. We selected this gene based on a recent report of ERK/E2F1 signaling in regulating RRM2 expression, dCTP pool and gemcitabine sensitivity [17]. The E2F1 rs3213150 polymorphism is an intronic and tag SNP selected in our study. Interesting, we observed significant association of this SNP with CR rate after Ara-C based induction therapy. In addition, a combined effect of the E2F1 rs3213150 and RRM2 rs1130609 polymorphisms on CR rate is observed. The CR rate decreased with the number of unfavorable genotypes carried at both loci $(66.7 \%$ in carriers of both favorable genotypes, $51.6 \%$ in carriers of one unfavorable genotype, and $16.6 \%$ in carriers of unfavorable genotypes for both SNPs), and unfavorable genotypes at both loci results in about ninefold increase in non-CR risk after Ara-C based chemotherapy $(\mathrm{OR}=9.780,95 \%$ CI $1.099-87.069, P=0.041)$. Our study suggest the importance of concomitant consideration of genetic variants of both $E 2 F 1$ and $R R M 2$ in evaluation of Ara-C response in AML patients.

$S A M H D 1$ is a recently identified ara-CTPase that can hydrolyze and detoxify intracellular ara-CTP [12]. Inhibition of the enzyme or CRISPR-Cas9-mediated disruption of SAMHD1 can sensitize AML cells to Ara-C [13]. In our study, we analyzed associations of three SAMHD1 SNPs (rs6102991 in the promoter, rs28372906 in 5'-UTR, and rs6029941 in 3'-UTR) with drug response and AML prognosis. We observed that carriers of the rs6102991 $\mathrm{G}$ allele showed a tendency with decreased risk of nonCR after Ara-C based therapy in a dominant model $(\mathrm{OR}=0.611, \quad 95 \%$ CI $0.369-1.013, \quad P=0.055)$. The rs6102991 polymorphism is located at -4395 upstream transcription start site (T-4395C). eQTL analysis indicates that the rs6102991 polymorphism results in decreased SAMHD1 mRNA expression, which supports our clinic findings. As the SNPs is far from the transcription start site, our findings indicate potential influence of rs6102991 or other variants in high LD with it on Ara-C response through affecting $S A M H D 1$ mRNA expression. Few studies have focused on functions of rare SAMHD1 coding variations on HIV infection and replication [33, 34], however, there is still a gap between $S A M H D 1$ variations and the clinical relevance. Because SNPs information for the SAMHD1 promoter is very limited in the SNP database available, further study is required to explore the potentially causative variant(s).

There were some limitations in our study. For instance, the outcomes of our study failed to undergo multiple test adjusting, some $P$ values lost statistical significance when Bonferroni correction was performed possibly due to limited sample size included in our study. In addition,
Ara- $\mathrm{C}$ response is affected by various genetic factors, the contribution of any unique gene in the drug response might be limited. And due to the limitations of the Massarray genotyping system, we failed to genotype some of the important SNPs such as RRM1rs1561876 [18]. For the AML patients involved in our study, genotype distribution for 4 SNPs (CMPK1 rs7543016, SAMHD1 rs28372906, RRM1 rs183484, and $R R M 2$ rs1130609) were not in Hardy-Weinberg equilibrium, these may partially be explained by the internal function of the genes in etiology of AML rather than in Ara-c response. We suppose that these polymorphisms may also act as susceptibility genes for AML, which may account for the abnormal distribution of the genotype for these SNPs in the AML patients. Of course, this should be confirmed in further study.

\section{Conclusions}

Our current study demonstrated that SNPs in genes involved in Ara-C metabolic pathway are associated with drug response to Ara- $C$ based induction therapy and prognosis of AML in Chinese patients, though further confirmatory studies and functional evaluations of the newly identified SNPs are needed to further support our findings. In addition, we suggest consideration of combined genotypes in the Ara-C metabolic pathway in evaluation of the associations between genetic variations and drug response in AML. Our findings provide insightful information for the understanding of individual difference in drug response and potential biomarkers for identification of patients with increased risk of chemoresistance or poor prognosis.

\section{Additional files}

Additional file 1: Table S1. Unconditional logistic regression analysis of clinical variables associated with non-CR risk in AML patients.

Additional file 2: Table S2. Multivariate Cox regression analysis of clinical factors influencing AML OS and RFS.

\footnotetext{
Abbreviations

Ara-C: cytarabine arabinoside; dNTP: deoxy-ribonucleoside triphosphate; AML: acute myeloid leukemia; ara-CTP: cytarabine arabinoside triphosphate; CR: complete remission; RFS: relapse-free survival; OS: overall survival; eQTL: expression Quantitative Trait Loci; SNPs: single nucleotide polymorphisms; SLC29A1: solute carrier family 29 member 1; DCK: deoxycytidine kinase; NDPKs: nucleoside diphosphate kinases; dCTP: deoxycytidine triphosphate; SAMHD1: SAM domain and HD domain 1; dATP: deoxyadenosine triphosphate; dTTP: deoxythymidine triphosphate; dGTP: deoxyguanosine triphosphate; RR: ribonucleotide reductase; CTP: cytidine triphosphate; RRM1: ribonucleotide reductase catalytic subunit $M 1 ; R R M 2$ : ribonucleotide reductase catalytic subunit M2; WT1: Wilms tumor 1; DNMT3A: DNA methyltransferase 3 alpha; E2F1: transcription factor 1; FLT3: FMS-like Tyrosine Kinase3; NMP1: nucleophosmin; NME1: nucleoside diphosphate kinase 1; NME2: nucleoside diphosphate kinase 2; EFS: event free survival; CMPK1: cytidine/uridine monophosphate kinase 1; EMR: electronic medical record; HSCT: haematopoietic stem cell transplantation; HWE: Hardy-Weinberg Equilibrium; FAB: French-Britain-American;
} 
WBC: white blood cell; LDH: lactate dehydrogenase; BM: bone marrow; RBC: red blood cell; Allo-SCT: allogeneic hematopoietic stem cell transplantation; CR: complete remission; THP: pirarubicin; MA: Ara-C + mitoxantrone; IA: Ara-C+ idarubicin; TA: Ara-C + pirarubicin; DA: Ara-C+ daunorubicin; CAG: Ara-C + aclarubicin + G-CSF; G-CSF: granulocyte colony-stimulating factor; OR: odds ratio; HR: hazard ratio; Cl: confidence interval; LD: linkage disequilibrium; HIV: human immunodeficiency virus.

\section{Authors' contributions}

ZKW made main contributions to read document and design research, specimen collection, sorting date, date analysis and drafting manuscript; CP, ZDY, YH and LYL were assisting in specimen collection and acquisition of clinical date; LH and CLN were mainly responsible for follow-up; and CXP provided substantial guidance and revised manuscript; CS and ZG given some useful advice; ZH, CSP, ZXL helped to collect specimen smoothly. All authors read and approved the final manuscript.

\section{Author details}

1 Department of Clinical Pharmacology, Xiangya Hospital, Central South University, Changsha 410078, People's Republic of China. ${ }^{2}$ Institute of Clinical Pharmacology, Central South University, Hunan Key Laboratory of Pharmacogenetics, Changsha 410078, People's Republic of China. ${ }^{3}$ National Clinical Research Center for Geriatric Disorders, Xiangya Hospital, Central South University, Changsha 410008, Hunan, People's Republic of China. ${ }^{4}$ Department of Hematology, Xiangya Hospital, Central South University, Changsha 410078, People's Republic of China. ${ }^{5}$ Department of Clinical Pharmacology, Xiangya Hospital, Central South University, Changsha 410008, Hunan, China.

\section{Acknowledgements}

The authors would like to thank Prof. Hui Zeng, Shu-ping Chen, Xie-lan Zhao for unselfish assistance with colleting specimen and acquiring clinical date.

\section{Competing interests}

The authors declare that they have no competing interests.

\section{Availability of date and materials}

The datasets supporting the conclusions of this article are included within the article (and its additional files).

\section{Consent for publication}

Not applicable.

\section{Ethics approval and consent to participate}

This study was approved by the Ethics Committee of Institute of Clinical Pharmacology, Central South University (Registration Number: CTXY-1200252). Clinical study admission (Registration Number: ChiCTR-PPC-14005297) was approved by the Chinese Clinical Trial Register and informed consent was obtained from each patient or their family members.

\section{Funding}

This project was supported by Chinese National Science Foundation (Nos. $81673518,81422052)$, the National key R\&D program (No. 2017YFC0909302).

\section{Publisher's Note}

Springer Nature remains neutral with regard to jurisdictional claims in published maps and institutional affiliations.

Received: 16 January 2018 Accepted: 27 March 2018

Published online: 10 April 2018

\section{References}

1. Estey E, Dohner H. Acute myeloid leukaemia. Lancet. 2006;368:1894-907.

2. Dohner H, Estey EH, Amadori S, Appelbaum FR, Buchner T, Burnett AK, et al. Diagnosis and management of acute myeloid leukemia in adults: recommendations from an international expert panel, on behalf of the European LeukemiaNet. Blood. 2010;115:453-74.

3. Wang JJ, Selawry OS, Vietti TJ, Bodey GP Sr. Prolonged infusion of arabinosyl cytosine in childhood leukemia. Cancer. 1970;25:1-6.
4. Kumar CC. Genetic abnormalities and challenges in the treatment of acute myeloid leukemia. Genes Cancer. 2011;2:95-107.

5. Boffo S, Damato A, Alfano L, Giordano A. CDK9 inhibitors in acute myeloid leukemia. J Exp Clin Canc Res. 2018;37:36.

6. Miyawaki S, Ohtake S, Fujisawa S, Kiyoi H, Shinagawa K, Usui N, et al. A randomized comparison of 4 courses of standard-dose multiagent chemotherapy versus 3 courses of high-dose cytarabine alone in postremission therapy for acute myeloid leukemia in adults: the JALSG AML201 Study. Blood. 2011;117:2366-72.

7. Wiley JS, Jones SP, Sawyer WH, Paterson AR. Cytosine arabinoside influx and nucleoside transport sites in acute leukemia. J Clin Investig. 1982;69:479-89.

8. Yamauchi T, Negoro E, Kishi S, Takagi K, Yoshida A, Urasaki Y, et al. IntracelIular cytarabine triphosphate production correlates to deoxycytidine kinase/cytosolic 5'-nucleotidase II expression ratio in primary acute myeloid leukemia cells. Biochem Pharmacol. 2009;77:1780-6.

9. Van Rompay AR, Johansson M, Karlsson A. Phosphorylation of nucleosides and nucleoside analogs by mammalian nucleoside monophosphate kinases. Pharmacol Ther. 2000;87:189-98.

10. Attwood PV, Muimo R. The actions of NME1/NDPK-A and NME2/NDPK-B as protein kinases. Lab Invest. 2018;98:283-90.

11. Crisp LB, Smith SM, Mathers MA, Young GA, Lyons SD, Christopherson RI. Effects of cytosine arabinoside on human leukemia cells. Int J Biochem Cell Biol. 1996;28:1061-9.

12. Schneider C, Oellerich T, Baldauf H, Schwarz SM, Thomas D, Flick R, et al. SAMHD1 is a biomarker for cytarabine response and a therapeutic target in acute myeloid leukemia. Nat Med. 2017;23:250-5.

13. Herold N, Rudd SG, Ljungblad L, Sanjiv K, Myrberg IH, Paulin CB, et al. Targeting SAMHD1 with the Vpx protein to improve cytarabine therapy for hematological malignancies. Nat Med. 2017;23:256-63.

14. Whelan J, Smith T, Phear G, Rohatiner A, Lister A, Meuth M. Resistance to cytosine arabinoside in acute leukemia: the significance of mutations in CTP synthetase. Leukemia. 1994;8:264-5.

15. Chimploy K, Tassotto ML, Mathews CK. Ribonucleotide reductase, a possible agent in deoxyribonucleotide pool asymmetries induced by hypoxia. J Biol Chem. 2000;275:39267-71.

16. Lamba JK. Genetic factors influencing cytarabine therapy. Pharmacogenomics. 2009;10:1657-74.

17. Xia G, Wang H, Song Z, Meng Q, Huang X, Huang X. Gambogic acid sensitizes gemcitabine efficacy in pancreatic cancer by reducing the expression of ribonucleotide reductase subunit-M2 (RRM2). J Exp Clin Cancer Res. 2017;36:107.

18. Cao X, Mitra AK, Pounds S, Crews KR, Gandhi V, Plunkett W, et al. RRM1 and RRM2 pharmacogenetics: association with phenotypes in HapMap cell lines and acute myeloid leukemia patients. Pharmacogenomics. 2013;14:1449-66.

19. Minkovsky A, Sahakyan A, Bonora G, Damoiseaux R, Dimitrova E, Rubbi L, et al. A high-throughput screen of inactive $X$ chromosome reactivation identifies the enhancement of DNA demethylation by 5-aza-2'-dC upon inhibition of ribonucleotide reductase. Epigenetics Chromatin. 2015;8:42.

20. Megías-Vericat JE, Montesinos P, Herrero MJ, Moscardó F, Bosó V, Martínez-Cuadrón D, et al. Influence of cytarabine metabolic pathway polymorphisms in acute myeloid leukemia induction treatment. Leuk Lymphoma. 2017;58:2880-94.

21. Yan $\mathrm{H}$, Zhang DY, Li X, Yuan $X Q$, Yang YL, Zhu KW, et al. Long non-coding RNA GAS5 polymorphism predicts a poor prognosis of acute myeloid leukemia in Chinese patients via affecting hematopoietic reconstitution. Leuk Lymphoma. 2017;58:1948-57.

22. Zhang DY, Yan H, Cao S, Zhang W, Li XL, Zeng H, et al. Wilms tumor 1 rs 16754 predicts favorable clinical outcomes for acute myeloid leukemia patients in South Chinese population. Leuk Res. 2015;39:568-74.

23. Zhang DY, Yuan XQ, Yan H, Cao S, Zhang W, Li XL, et al. Association between DCK $35708 \mathrm{~T}>\mathrm{C}$ variation and clinical outcomes of acute myeloid leukemia in South Chinese patients. Pharmacogenomics. 2016;17:1519-31.

24. Yuan $X Q$, Zhang DY, Yan $H$, Yang YL, Zhu KW, Chen YH, et al. Evaluation of DNMT3A genetic polymorphisms as outcome predictors in AML patients. Oncotarget. 2016;7:60555-74.

25. Zhu GZ, Yang YL, Zhang YJ, Liu W, Li MP, Zeng WJ, et al. High expression of AHSP, EPB42, GYPC and HEMGN predicts favorable prognosis in FLT3-ITDnegative acute myeloid leukemia. Cell Physiol Biochem. 2017;42:1973-84. 
26. Yuan XQ, Peng L, Zeng WJ, Jiang BY, Li GC, Chen XP. DNMT3A R882 mutations predict a poor prognosis in AML: a meta-analysis from 4474 patients. Medicine (Baltimore). 2016;95:e3519.

27. Braunagel D, Schaich M, Kramer M, Dransfeld C, Ehninger G, Mahlknecht U. The T_T genotype within the NME1 promoter single nucleotide polymorphism $-835 \mathrm{C} / \mathrm{T}$ is associated with an increased risk of cytarabine induced neurotoxicity in patients with acute myeloid leukemia. Leuk Lymphoma. 2012;53:952-7.

28. Horak CE, Lee JH, Elkahloun AG, Boissan M, Dumont S, Maga TK, et al. Nm23-H1 suppresses tumor cell motility by down-regulating the lysophosphatidic acid receptor EDG2. Cancer Res. 2007:67:7238-46.

29. Qu S, Long J, Cai Q, Shu XO, Cai H, Gao YT, et al. Genetic polymorphisms of metastasis suppressor gene NME1 and breast cancer survival. Clin Cancer Res. 2008;14:4787-93.

30. Yokoyama A, Okabe-Kado J, Sakashita A, Maseki N, Kaneko Y, Hino K, et al. Differentiation inhibitory factor $\mathrm{nm} 23$ as a new prognostic factor in acute monocytic leukemia. Blood. 1996:88:3555-61.
31. Okabe-Kado J, Kasukabe T, Honma Y, Kobayashi H, Maseki N, Kaneko Y. Extracellular NM23 protein promotes the growth and survival of primary cultured human acute myelogenous leukemia cells. Cancer Sci. 2009;100:1885-94.

32. Cao HX, Miao CF, Yan L, Tang P, Zhang LR, Sun L. Polymorphisms at microRNA binding sites of Ara-C and anthracyclines-metabolic pathway genes are associated with outcome of acute myeloid leukemia patients. J Transl Med. 2017; 15:235

33. Rentoft M, Lindell $K$, Tran $P$, Chabes AL, Buckland RJ, Watt DL, et al. Heterozygous colon cancer-associated mutations of SAMHD1 have functional significance. Proc Natl Acad Sci USA. 2016;113:4723-8.

34. He DD, Mao SL, Wang W, Jin YB, Zhang HL, Ling F. SAMHD1 polymorphisms were significantly correlated with HIV/SIV virus load in PBMC from Chinese rhesus macaques and cynomolgus macaques. Curr HIV Res. 2017;15:15-22.

\section{Submit your next manuscript to BioMed Central and we will help you at every step:}

- We accept pre-submission inquiries

- Our selector tool helps you to find the most relevant journal

- We provide round the clock customer support

- Convenient online submission

- Thorough peer review

- Inclusion in PubMed and all major indexing services

- Maximum visibility for your research

Submit your manuscript at www.biomedcentral com/submit 